\title{
Zur Frage nach Veränderung: Erfahrungsblitzlichter steirischer Krankenhausseelsorger*innen
}

\author{
Sabine Petritsch
}

Wenn man ein Kleinkind beim Bauen neuer Welten beobachtet, staunt man: Es wird ein Bauwerk mit vielen Türmen und Gängen errichtet. Tiere und menschliche Figuren drängen sich hindurch. Das Kleinkind ist ,fertig'. Es betrachtet sein Werk von allen Seiten. Was nimmt es wahr? Die Größe und Schönheit? Die Buntheit? Es lächelt und freut sich. Als es seine Perspektive ändert, kommt es zum Entschluss, das Bauwerk gehört geändert. Ärger und Zorn werden ausgedrückt. Es lernt: genaues Hinschauen löst unterschiedliche Emotionen und Ambivalenzen aus. Mehrperspektivisches Betrachten ermöglicht zwar, mehrere Facetten wahrzunehmen, es geht allerdings auf Kosten von Eindeutigkeit.

Um mehrperspektivische Betrachtungsweisen geht es in diesem Beitrag: Es kommt zur Sprache, welche Veränderungen Vertreter*innen der römisch-katholischen Krankenhausseelsorge Steiermark wahrnehmen und inwiefern diese die Seelsorge betreffen. In einem Austauschtreffen der verantwortlichen Teamleitungen in den Krankenhäusern der Steiermark wurde über Veränderungen durch Corona bei liturgischen Feiern, beim Einsatz Ehrenamtlicher, der Stellung der Seelsorge und bei Kindern und Jugendlichen berichtet. Aber wie sehr hat sich tatsächlich etwas verändert? Insgesamt elf Krankenhausseelsorger"innen bzw. Priester, an unterschiedlichen Standorten und bei verschiedenen Trägern in der Steiermark tätig, haben mir schriftlich auf Fragen zu jeweils einem Themenschwerpunkt geantwortet, um ihre Wahrnehmungen von Veränderung mit mir und Ihnen allen, die den Beitrag lesen, zu teilen. ${ }^{1}$ Es sind unterschiedliche

1 Die Themenschwerpunkte sind: Ehrenamt, Liturgie, Kinder/Jugendliche, Offenes und Grundsätzliches; zu diesen stellte ich schriftlich drei Fragen an mindestens zwei verschiedenen Personen und erhielt dazu Antworten. Die Manuskripte von Johannes Biener, Bert Brottrager, Michaela Fetz, Michaela Hirzer-Weiß, Markus Kern, Manuela Krtek, Petra Lackner-Haas, Erwin Löschberger, Hans Mosbacher, der als Priester die Sakramente im LKH Mürzzuschlag spendet, aber kein Krankenhausseelsorger ist, Anita Ulz und Friedrich Weingartmann liegen bei mir auf. Ihre Perspektiven kommen in Form wörtlicher Zitate in diesem Essay zu Wort. 
persönliche Bilder und Erfahrungen, die wir als steirische Krankenhausseelsorger*innen anbieten.

\section{Der Unterschied von einem Jahr'2: Grundsätzliche Beobachtungen}

Welche Unterschiede nehmen Seelsorger*innen nun wahr? Neben den Schutzmaßnahmen und dem Besuchsverbot, von dem Krankenhausseelsorger*innen weitgehend ausgenommen sind, zeigt sich, dass vor allem auch allgemein gesellschaftliche Themen die im Gesundheitswesen Tätigen herausfordern. Bert Brottrager, Krankenhausseelsorger im LKH Hochsteiermark Standort Leoben, schreibt dazu: „Während vor einem Jahr noch das Thema Schutzkleidung und -maßnahmen einen großen Stellenwert einnahm, ist es nun für viele Kolleginnen das ständige On/Off der Schulen und Kinderbetreuungsstätten." Auch Manuela Krtek, Krankenhausseelsorgerin am LKH Hochsteiermark Standort Bruck/Mur, nimmt diesbezüglich starke Veränderungen wahr: „Die Sorge um vertraute Menschen hat viele dazu bewogen, Kontakte auch außerhalb des Arbeitsfeldes bewusst auf ein minimales $\mathrm{Maß}$ zu reduzieren, das Lebensumfeld noch einmal stärker einzuschränken.“ Zu Änderungen im privaten Umfeld kommen jene im Arbeitsalltag dazu, wenn Abläufe aufgrund von Erkrankungen des Personals zu Umschichtungen führen, geplante Operationen verschoben werden etc. Gleichzeitig nehmen wir Krankenhausseelsorger"innen wahr, wie eine größer werdende Solidarität innerhalb des

Die Krankenhausseelsorger*innen sind an folgenden unterschiedlichen steirischen Standorten tätig: Ordensspitäler der Elisabethinen und Kreuzschwestern in Graz, LKH-Universitätsklinikum Graz, LKH Graz 2 (Standort West), LKH Hochsteiermark, LKH Mürzzuschlag, LKH Feldbach sowie Unfallkrankenhaus AUVA Graz. Dadurch sollen verschiedene Perspektiven von Stadt und Land, unterschiedliche Zugänge der Trägerschaften etc. sichtbar werden. Gleichzeitig bin auch ich selbst als Endverfasserin nicht ausgenommen von den eigenen Erfahrungen und Deutungen, die auch mir als Krankenhausseelsorgerin am LKH-Universitätsklinikum Graz begegnen; sie spiegeln sich in den Fragen, der Auswahl und letztendlich der Komposition.

2 Der Unterschied von einem Jahr bildet sich in den Manuskripten mit den Fragen zum Themenbereich Offenes/Grundsätzliches ab. Dazu wurden folgende Fragen gestellt: „Gibt es Gefühle, Themen, Anliegen, die du in der Zeit stärker wahrnimmst und mit denen du öfters konfrontiert wirst als früher? Was hat sich im gesamten System Krankenhaus aus deiner Sicht verändert? Wenn es eine gute Fee gibt, welche Wünsche soll sie welchen Stellen übermitteln?“ Manuela Krtek und Bert Brottrager wurden dazu befragt. 
Personals entsteht - auch mit Seelsorger*innen. Darüber hinaus haben andere Themen an Relevanz gewonnen, wie Manuela Krtek schreibt:

„Das Thema der Kommunikation ist noch bedeutender geworden. Maßnahmen mussten erarbeitet, kommuniziert und laufend angepasst werden. Neue Formate für Besprechungen wurden entwickelt. Sowohl innerhalb des gebildeten Krisenstabes als auch auf allen anderen Ebenen ist die Bedeutung der interprofessionellen Zusammenarbeit gestiegen.“

Zum Dienst der Krankenhausseelsorger*innen gehört einerseits in Kontakt mit den Mitarbeiter"innen zu sein, andererseits vor allem mit den Patient*innen. Manuela Krtek beschreibt Veränderungen:

„In einer Zeit, in der Besuchsmöglichkeiten stark eingeschränkt waren, hatte das Angebot der Krankenhausseelsorge noch mehr Bedeutung. Noch einmal verstärkt wurden eigene Erfahrungen mit Krisensituationen aus der Biographie, aber auch die Belastungen in Hinblick auf die Pandemie, die Erfahrung von Einsamkeit durch die reduzierten Kontaktmöglichkeiten angesprochen.“

Die Corona-Pandemie hat auch zur Klärung der Frage nach der Stellung der Seelsorge beigetragen:

„Wer ist für unsere Sicherheit verantwortlich? Wer entscheidet, ob und wie wir auch in Covid-Zeiten für das Personal und für die Patienten da sein können? Welche Regeln und Maßnahmen gelten für unser Tun? Die Notwendigkeit der Klärung dieser Fragen hat zu einer exakteren Definition geführt, wie wir zum Haus gehören, wer für uns verantwortlich, Ansprechpartner und Weisungsgeber ist“

- so Manuela Krtek. Durchwegs lässt sich feststellen, dass Seelsorge als wichtiger und verlässlicher Partner im Gesundheitswesen gesehen wird, sowohl für Patient"innen als auch für Mitarbeitende.

Erstes Zwischenfazit: Von gesellschaftlichen Brennpunktthemen wie z. B. Vereinbarkeit von Familie und Beruf, die Reduzierung von privaten Kontakten etc. sind Mitarbeitende im Gesundheitswesen nicht ausgenommen. Für die Seelsorge zeigt sich, dass die Corona-Pandemie dazu geführt hat, dass die Krankenanstalten ihr Verhältnis zur Seelsorge klärten. 


\section{Nichts geht verloren - zur Frage des Ehrenamtes ${ }^{3}$}

Die Krankenhausseelsorge lebt vom ehrenamtlichen Engagement. Gut ausgebildete Frauen und Männer bringen sich freiwillig in den Dienst der Krankenhausseelsorge ein. Eine Wahrnehmung ist, dass das Besuchsverbot in Zeiten der Corona-Pandemie das Ehrenamt stark betrifft und dadurch deutlich wird, wie Krankenanstalten das Ehrenamt sehen. Nach dem ersten harten Lockdown im März 2020 erfolgte ein erstes Aufatmen durch die Lockerung des strengen Verbots ab Sommer 2020. Anita Ulz beschreibt ihre Erfahrungen als Krankenhausseelsorgerin im LKH Feldbach:

„Ab ersten Juli war es dann für Ehrenamtliche wieder möglich, Kranke zu besuchen, sie sakramental zu begleiten und auch den Gottesdienst zu feiern. Die Spannung, die wir in einer Teamsitzung außerhalb des Krankenhauses vernahmen, war deutlich, denn jetzt wurden die Seelsorgenden, wie auch alle anderen im LKH Arbeitenden, reglementiert: Masken tragen, Hände desinfizieren, Abstand halten. Die gesellschaftliche Spannung in der Pro- und Contra-Haltung, die Regeln der Bundesregierung zu befolgen, zeigte sich auch spürbar bei den Ehrenamtlichen: war es zuvor der gemeinschaftlich anerkannte Schutz der Patient*innen und Angestellten, traten jetzt individuelle Themen zutage: die Angst, sich mit dem Virus im LKH zu infizieren, die Freude wieder Gespräche zu führen sowie der allgemeine Widerstand gegen das Tragen der Maske.“

Nach dem Sommer ist es erneut zu Einschränkungen gekommen, die u.a. das LKH Feldbach betroffen haben:

„Im Herbst 2020 folgte dann wieder der völlige Ausschluss von Ehrenamtlichen im Krankenhaus, sowohl in der Seelsorge, als auch im Hospizteam. [...] Die gewohnte ,Normalität ${ }^{\star}$ war verschwunden und der Kontakt der Hauptamtlichen zu den [Ehrenamtlichen, Ergänzung S.P.] ist bis zum jetzigen Zeitpunkt nur mehr per Telefon oder Mail aufrecht zu erhalten.“

3 Folgende Fragen wurden zum Bereich des Ehrenamtes den Krankenhausseelsorger*innen Anita Ulz (LKH Feldbach) und Erwin Löschberger (AUVA UKH Graz, LKH Graz 2) gestellt: „Wie ist es Ehrenamtlichen aktuell möglich, tätig zu sein? Nimmst du einen Unterschied in der Begleitung wahr? Was glaubst du, dass Ehrenamt in der KHS für die Zukunft bedeutet?" 
Anders ist es im Unfallkrankenhaus Graz, so Krankenhausseelsorger Erwin Löschberger:

„Im UKH-Graz gibt es eine ehrenamtliche Mitarbeiterin in der Seelsorge. Nach dem Aussetzen der Tätigkeit während des ersten Lockdowns im März 2020 konnte sie im Juni 2020, wie wir Hauptamtlichen auch, wieder den Dienst aufnehmen. Sie wird seit Dezember 2020 wöchentlich vor Beginn ihrer seelsorglichen Besuche getestet und ist selbstverständlich zum Tragen einer FFP2-Maske verpflichtet. Im LKH 2, Standort Süd, ist derzeit keine ehrenamtliche Mitarbeiterin aktiv. Der Grund liegt einerseits in der persönlichen Vorsicht der ehrenamtlichen Mitarbeiter*innen, andererseits in der noch nicht erfolgten Impfung. Nach erfolgter Impfung - die in Aussicht gestellt wurde wollen zwei Mitarbeiter*innen den Besuchsdienst wieder beginnen. Alle ehrenamtlichen Dienste, die mit gottesdienstlichen Feiern zu tun haben, sind bis zum heutigen Tag nicht möglich.“

In der Begleitung Ehrenamtlicher durch Hauptamtliche sind nun andere Themen vorrangig. Anita Ulz beschreibt, dass die eigene Innerlichkeit stärker in den Fokus des Gesprächs rückt:

„Neben dem plötzlichen Verlust der Umarmungen, der Tiefe der persönlichen Gespräche untereinander, der persönlichen Erfahrungen, die auch Raum im Miteinander hatten, wurde auch der Verlust der persönlichen Bereicherung in der Ausübung des Dienstes für andere, im ehrenamtlichen Besuchsdienst besprochen."

Das Anliegen, seelsorglich tätig zu sein, war dennoch für viele Ehrenamtliche wichtig, und Anita Ulz schildert, dass ehrenamtliche Krankenhausseelsorger*innen andere Wege suchten:

„Es war uns wichtig, bewusst auf Begegnungen bei Spaziergängen zu achten und dort, wachsam hörend, wie eben im gewohnten LKHDienst, Menschen ihr Ohr zu leihen, sie zu ermutigen und aufzurichten. Einer Ehrenamtlichen gelang es in großer Kreativität und Lebensfreude in ihrem Ort Menschen zum persönlichen Karwochenweg zu ermutigen. Für rund 70 Haushalte fertigte sie mit ihrer Familie Impulse, Brotlaibe und Gedanken und legte sie vor die Tür. Es gelang ihr, das Evangelium in die Häuser zu tragen und das Herz der Menschen unaufdringlich zu berühren."

Sie spricht vom „Lernort Krankenhaus“, der „aufgrund der Dichte der Erfahrungen und Authentizität der Menschen Ausgangspunkt für die Tiefe der Begegnungen in den Pfarren ist. Nicht zuletzt ermutigte auch die Be- 
gleitung und ehrenamtliche Ausbildung in der Diözese, ihre Kompetenzen weiterhin außerhalb des Krankenhauses einzusetzen."

Zweites Zwischenfazit: In vielen Krankenhäusern ist der ehrenamtliche Dienst nicht möglich. Dennoch lässt sich eine Prognose für die Zukunft des Ehrenamtes in der Krankenhausseelsorge nach der pandemischen Corona-Zeit aus den Erfahrungen nicht ableiten. Festzustellen ist, dass die Mehrheit der Ehrenamtlichen ihren Dienst zwar nicht im Krankenhaus ausübt, ihre erworbenen Fertigkeiten an anderen Orten fruchtbar werden.

\section{Individueller und flexibler - Feier der Liturgie}

Die Feier von Gottesdiensten und Ritualen, die Spendung von Sakramenten und Sakramentalien angesichts der Krankheit gehören wesentlich zur Krankenhausseelsorge. Auch unter den jetzigen Bedingungen ist dies mit erforderlichen Schutzmaßnahmen möglich. Dennoch haben sich Feiern, vor allem gemeinschaftliche, liturgische Feiern, in dieser pandemischen Zeit verändert. Das trifft sowohl Priester, als auch Frauen und Männer in der Krankenhausseelsorge. Darüber berichten drei ordinierte und zwei nicht ordinierte Seelsorger*innen. Zugleich denken sie über die Zukunft gottesdienstlicher Feiern im Kontext der Krankenhausseelsorge nach. ${ }^{4}$ Friedrich Weingartmann, Pfarrer und Leiter der Krankenhausseelsorge am LKH Feldbach, berichtet, dass es wieder möglich ist,

„jede Woche am Freitagabend die Hl. Messe zu feiern, allerdings ohne die persönliche Präsenz der Patient*innen. Eine Krankenhausseelsorgerin übernimmt die musikalische Gestaltung der Hl. Messe. Die Hl. Messe wird in die Krankenzimmer übertragen, sodass die Patient"innen im Krankenbett den Gottesdienst hören und mitfeiern können. Dieses liturgische Angebot wird dankbar angenommen."

4 Folgende Personen wurden befragt: Ein Priester des Universitätsklinikums Graz, der anonym bleiben möchte (im Text als Anonymous bezeichnet), Friedrich Weingartmann, Leiter der Krankenhausseelsorge am LKH Feldbach, Krankenhausseelsorgerin Michaela Hirzer-Weiß von den Ordensspitälern der Elisabethinen und Kreuzschwestern Graz. Hans Mosbacher, der dem LKH Mürzzuschlag zugeordnet ist, Sakramente spendet und die Hl. Messe feiert, hat gemeinsam mit der Leiterin der Krankenhausseelsorge am LKH Mürzzuschlag Michaela Fetz, folgende Fragen reflektiert: „Gibt es eine Veränderung, die am meisten spürbar ist in der Liturgie? Inwiefern ist eine Veränderung durch die Auflagen und Maßnahmen etc. für das Team spürbar? Für wen mehr, für wen weniger? Was wird in Zukunft an Bedeutung in der rituellen Begleitung von Menschen in der KHS gewinnen?“ 
Die Pfarre am LKH Universitätsklinikum Graz ist in dieser Hinsicht eine Ausnahme; sie ist eine frei stehende Pfarrkirche, in der, unter Einhaltung der Maßnahmen, Gottesdienst mit Patient*innen, Angehörigen, Mitarbeiter*innen sowie durch Übertragung auch am Krankenbett gefeiert wird. Jedoch sind Gottesdienste auf Stationen nicht mehr möglich. Anonymous am LKH Universitätsklinikum berichtet:

„Für mich am stärksten spürbar war vielleicht das Wegfallen des liturgischen Gesanges. [Gleichzeitig haben] die anderen, eigentlich als verbindlich vorgesehenen und leider oft vernachlässigten, ,Orte' der Stille und des Schweigens in der Liturgie - wie etwa die Stille zwischen Gebetseinladung und Oration - wieder an Bedeutung und Intensität gewonnen. [Ein] leichter Rückgang [ist] von Kommunionwünschen auf den Stationen überhaupt zu spüren.“

Dazu Michaela Hirzer-Weiß, Seelsorgerin in den Ordensspitälern der Elisabethinen und Kreuzschwestern in Graz:

„Es gab/gibt die Anweisung für Patient*innen, ihr Zimmer/ihre Station möglichst nicht zu verlassen, die je nach Phase der Pandemie unterschiedlich streng gehandhabt wurde. Jedenfalls gibt es ein Bedürfnis von Patient*innen, die Krankenhauskapelle auch für sich alleine zu nutzen, zum Rückzug in die Stille, zum Gebet.“

Ein gemeinsames Ringen, auch im Team der Seelsorger*innen, ist erforderlich, wenn es um Berührungen innerhalb liturgischer Feiern geht, wie Michaela Hirzer-Weiß schildert:

„Diskussionen hatten wir z. B. bei den Themen ,Aschenkreuz austeilen' oder ,Krankensegen anbieten'. Inwieweit sind Haut-zu-Haut-Berührungen seelsorglich, notwendig? Welche Unterschiede, sowohl seelsorglich gesehen als auch in der Stationskultur, gibt es auf den verschiedenen Stationen, von der Palliativstation bis zur Chirurgie?“

Ich selber nehme wahr, dass ein Abwägen unterschiedlicher Komponenten und Sichtweisen auch mit dem Pflegepersonal nötig ist, um zu einer gemeinsamen guten Entscheidung zu kommen, was möglich ist und was vermieden werden soll. Letztlich geht es um die konkrete Person, um den Kranken und sein Umfeld. Das erfordert manchmal Kreativität. Michaela Fetz und Pfarrer Hans Mosbacher, LKH Mürzzuschlag, berichten:

„Bald nach dem Frühjahr 2020 wurde auf Anfrage von Kranken bzw. Angehörigen auch wieder die Krankensalbung auf allen Stationen möglich. Hier wurde und wird auch vom Priester Offenheit und Krea- 
tivität gefordert, z. B. wurden über ,Whatsapp“ isolierte Kinder und Enkelkinder zur Krankensalbung dazu geholt und auch eingebunden; die Dankbarkeit der Angehörigen war groß.“

Neben Abwägungs- und Entscheidungsprozessen hinsichtlich dessen, hat sich auch sonst viel da und dort geändert: Der Wegfall der Feier des Gottesdienstes in den Kapellen vor Ort, der besonders Priester betrifft, hat beispielsweise im LKH Mürzzuschlag bei Michaela Fetz zur Folge,

„dass der Tätigkeitsbereich von Seelsorger*innen mehr als früher auch in den rituell-liturgischen Bereich ging und geht, da immer wieder Menschen äußern, dass sie eine Kommunionfeier wünschen. [...] Auch Segensfeiern wurden vermehrt gewünscht (hier ist zu beachten, dass die kirchlich-sakramentale Einbindung der Menschen im Mürztal oft gering ist).“

Gleiches beobachtet Michaela Hirzer-Weiß in beiden Grazer Ordensspitälern und vermutet, dass das auch künftig so bleiben wird. Denn „die Patient*innen mögen sich auch nicht alle gerne im Morgenmantel, mit dem Rollator, den Gehhilfen, dem Urinsackerl etc. in der Öffentlichkeit zeigen, vor allem, wenn der Weg zur Kapelle etwas weiter ist." Auch wenn einige ältere Patient*innen Kommunionfeiern wünschen, zeigt sich, so Michaela Hirzer-Weiß, folgendes:

„Menschen können sich unter rituellen Angeboten oft nichts vorstellen. Die Vermittlung und Erklärung finde ich nicht ganz einfach. Wahrscheinlich braucht es auch die Ausarbeitung von neuen Ritualen, z. B. einen speziellen Segen vor einer OP oder für Angehörige; oder ein Dankbarkeitsritual, wenn etwas gut gegangen ist.“

Michaela Fetz und Hans Mosbacher resümieren: „Für die Zukunft wird [...] die rituelle Begleitung mehr in den kleineren Rahmen der Zimmer wandern und damit persönlicher, individueller und kreativer werden und manchmal auch Verkündigung für die Mitkranken sein“; und Anonymous vom Universitätsklinikum Graz meint dazu: ,[S]owohl aus meiner Erfahrung als Seelsorger als auch als Patient [möchte ich] ein Plädoyer für ,liebevolle Einfachheit" und Schlichtheit abgeben. Weniger ist mehr."

Drittes Zwischenfazit: In der Liturgie scheint die Corona-Pandemie eine Veränderung für die Zukunft auszulösen: Kreatives, individuelles und wohl auch intimes Feiern im Rahmen eines Krankenhaus-Aufenthaltes scheint auch zukünftig gefragt sein, dem auch nicht priesterliche Seelsorger*innen vorstehen. 


\section{Kinder und Jugendliche eint die Konfrontation mit der Möglichkeit des Todes} mit der Welt ${ }^{5}$

„Corona provoziert, weil die Erkrankung zum Tode führen kann, und das ist bei den Onko-Kindern und Familien immer der Fall, das eint uns jetzt mit der Gesellschaft", stellt Markus Kern fest, Seelsorger an der Pädiatrisch-Hämatologischen Onkologie am Universitätsklinikum Graz. „Die Tabuthemen Sterben und Tod sind für schwer erkrankte Kinder und Jugendliche und deren Familien immer präsent. Insofern ist hier keine größere Veränderung spürbar. Inwiefern Ängste durch Corona verschärft worden sind, ist nicht eins zu eins zu sagen, diese Frage ist für mich offen.“

Es ist anzumerken, dass es zu einer deutlichen Reduzierung von Besuchsmöglichkeiten gekommen ist, sowohl von Begleitpersonen, als auch von ehrenamtlichen Vereinen, wie z. B. den „Gelben Tanten“, den „CliniClowns“ etc. Dies wirkt sich insofern aus, als Kinder, Jugendliche und ihre Familien dadurch weniger ,Programm‘ und mehr Zeit haben. Über Auswirkungen dieser Reduktion lässt sich nur spekulieren. Petra LacknerHaas, Seelsorgerin am Pädiatrischen Kinderzentrum am Universitätsklinikum Graz, berichtet wiederum von verstärkt aufkommenden Ängsten bei den Betroffenen:

„In meinen Begleitungen nehme ich einerseits Angst wahr. Angst, dass jemand aus den Helfersystemen das Virus bringen könnte. Familien mit schwer erkrankten Kindern, die ihre Kinder zuhause betreuen, sind auf Hilfsdienste, die teilweise täglich nach Hause kommen, angewiesen. Wenn diese wegfallen, ist das für die Familien sehr schwer handhabbar. Wenn diese kommen, bedeutet das ein vermehrtes Risiko einer möglichen Ansteckung. Hier gibt es oft große Unsicherheiten, Ängste und Sorgen. Eltern, vor allem Mütter, waren und sind in Zeiten von Corona noch stärker gefordert und belastet, vor allem wenn ihre Kinder schwer- und lebensbedrohlich erkrankt sind. Ihnen fehlt oft die Kraft und auch die Möglichkeit auf ihre Situation, auf ihr Leid, aufmerksam zu machen und leider auch die Lobby dafür.“

Kranke Kinder scheinen auch in nicht-pandemischer Zeit für die Mehrheit der Gesellschaft kaum Thema zu sein. Dabei zeigt sich, dass die Pandemie

5 Vom LKH Universitätsklinikum wurden zur Frage der Seelsorge bei Kindern und Jugendlichen Petra Lackner-Haas und Markus Kern wie folgt befragt: „Was nimmst du verstärkt bei Kindern, Jugendlichen, Familien wahr? Gibt es einen Unterschied in der Begleitung zur Zeit vor Corona? Gibt es Themen, die aus deiner Sicht gesellschaftspolitisch relevant sind?“ 
hier sogar etwas ermöglicht, was ungewöhnlich klingt: Eine Form von Gleichberechtigung zwischen kranken und gesunden Kindern. Kranke Kinder erleben in gewissem Maße das Gefühl von ,wir sind den anderen, gesunden Kindern gleich'! Denn: „,Onko Kinder' müssen Maske tragen. ,Onko Kinder' wissen was es heißt, Homeschooling zu haben, wie auch ihre Familien", gibt Markus Kern zu bedenken.

Viertes Zwischenfazit: In der Corona-Pandemie kommt unserer Wahrnehmung nach weder das Wohl gesunder und noch weniger das Wohl kranker Kinder zu Wort, geschweige denn ein Austausch dieser beiden Lebenswelten.

\section{Zusammenschau}

Was bedeuten diese Wahrnehmungen nun für die eingangs gestellte Frage: Inwiefern hat die Corona-Pandemie in der Seelsorge Veränderungen ausgelöst? Die Antwort fällt ambivalent aus. Erhöhte Schutzmaßnahmen, reduzierte Besuchsmöglichkeit, Verzicht auf gemeinschaftliche Gottesdienstfeiern etc. haben die Welt auch innerhalb des Krankenhauses verändert - aber auch in mancher Hinsicht nicht. Wenn ich als Krankenhausseelsorger"in mit dem/der Kranken in Kontakt trete, es zu einem Gespräch kommt, dieses in einer Begegnung mündet und sich möglicherweise sogar in einer Feier ausdrückt, dann ereignet sich etwas außerhalb von Zeit und Raum. Die Pandemie wird von einem größeren Geheimnis durchbrochen. Sie rückt zurück.

Die zur Verfügung gestellten Wahrnehmungen geben ein buntes, uneindeutiges, vielleicht sogar ambivalentes Bild von Krankenhausseelsorge der steirischen Kirche wieder. Wahrnehmungen entziehen sich den Kategorien von richtig und falsch, und doch sind sie wertvoll und so mancher Ausgangspunkt für Veränderungen. Das Krankenhaus kann als Lernort für die Gesellschaft gesehen werden: Von der Frage, welche Maßnahmen schützen, bis hin zu Gruppen, die übersehen werden, wie kranke Kinder und Jugendliche. Gleichzeitig entkommt man den Themen Krankheit, Tod, Angst, Verwundbarkeit, Einsamkeit und Zurückgeworfensein nicht - aber auch der Hoffnung nicht. Hoffnung, dass ,es ${ }^{6}$ schaffbar ist, wie auch die Pandemie bewältigbar ist, wenn wir merken, wir sind alle im gleichen Boot. Diese Solidarität wurde nicht zuletzt sichtbar im Tanz von hunderten Mitarbeiter*innen des LKH Universitätsklinikums Graz 
zu ,Jersualema ${ }^{6}$. Dieser Jerusalema Challenge schlossen sich viele weitere Kliniken an. Vielleicht entstehen ganz neue Bauwerke, wenn wir diesem Takt und Geist folgen - ein neues Miteinander, ein aufeinander Hören, wo Wahrnehmungen von Verschiedenheit Früchte tragen...

6 Siehe dazu: https:/www.youtube.com/watch?v=wmffLZnc3X8\&t=12s\&ab_channe l=LKH-Univ.KlinikumGraz [15. 05. 2021]. 
